\title{
AVALIAÇÃO DOS ÍNDICES DE EXTREMOS CLIMÁTICOS NO AGRESTE CENTRAL DE PERNAMBUCO
}

\section{EVALUATION OF CLIMATE EXTREME INDICES IN CENTRAL PERNAMBUCO}

Maendra Pollinne Arcoverde Soares ${ }^{1}$, Werônica Meira de Souza ${ }^{2}$, Ricardo Brauer Vigoderis ${ }^{3}$

${ }^{1}$ Graduanda do curso de Agronomia UAG/UFRPE, Garanhuns - PE, e-mail: meandra.arcoverde@gmail.com; ${ }^{2}$ Professora da UAG/UFRPE, Garanhuns-PE, e-mail: weronicameira@gmail.com; ${ }^{3}$ Professor da UAG/UFRPE, Garanhuns-PE, e-mail: vigoderis@ hotmail.com.

DOI: https://doi.org/10.52719/bjas.v1i2.2818

\section{RESUMO}

Vários fatores ocasionam modificações no clima, causando o surgimento de eventos que alteram o regime de chuvas e comprometendo o desenvolvimento da vida no planeta. Portanto, o objetivo do presente trabalho foi avaliar as tendências climáticas dependentes da precipitação pluviométrica no Agreste Central de Pernambuco por meio dos índices climáticos. Foram selecionados dados de precipitação pluviométrica de 9 dos 71 municípios que formam o Agreste de Pernambuco, sendo estes Alagoinha, Barra de Guarabira, Belo Jardim, Bezerros, Brejo da Madre de Deus, Cachoeirinha, Caruaru, Gravatá e São Bento do Una, no período de 1963 a 2017, obtidos no site da Agência Pernambucana de Águas e Clima (APAC). Os dados foram processados através do software RClimdex, e gerados os índices climáticos precipitação total anual, dias consecutivos secos, quantidade máxima de precipitação em um dia e número de dias com precipitação acima de $25 \mathrm{~mm}$. Os resultados parciais mostram uma tendência de diminuição da precipitação total anual para todas as localidades exceto Barra de Guabiraba e Cachoeirinha. Para os dias consecutivos secos observou-se um equilíbrio entre as localidades. Barra de Guabiraba, Cachoeirinha, Gravatá e Caruaru, apresentaram diminuição desse parâmetro. Quanto à quantidade máxima de precipitação em um dia, somente as cidades de Brejo da Madre de Deus e Cachoeirinha indicaram leves tendências negativas não significativas, as demais apontaram diminuição. Números de dias com precipitação acima de $25 \mathrm{~mm}$ apresentaram tendências negativas para todas as cidades, porém, valores não significativos. Mesmo que uma parte dos parâmetros avaliados não indicarem tendências significativas, pode-se afirmar que há uma diminuição na quantidade de chuva para região prejudicando assim toda forma de desenvolvimento da região, sobretudo agricultura e pecuária, que predomina na região, e que a falta de água impossibilita sua realização.

Palavras-chave: Parâmetros. Pluviometria. Software 


\section{ABSTRACT}

Several factors cause changes in the climate, causing the appearance of events that alter the rainfall regime, compromising the development of life on the planet. Therefore, the objective of the present work was to evaluate the climatic trends dependent on rainfall in the Central Agreste of Pernambuco from the climatic indexes. Rainfall data were selected from 9 of the 71 municipalities that form the Agreste of Pernambuco, being these Alagoinha, Barra de Guarabira, Belo Jardim, Bezerros, Brejo da Madre de Deus Cachoeirinha, Caruaru, Gravatá and São Bento do Una, during the 1963 to 2017, obtained on the website of the Pernambuco State Agency for Water and Climate (APAC). The data were processed through the software RClimdex, and the climatic indexes were generated annual total rainfall, consecutive dry days, maximum amount of precipitation in one day and number of days with rainfall above $25 \mathrm{~mm}$. The partial results show a tendency of decrease of total annual precipitation for all the localities except Barra de Guabiraba and Cachoeirinha. For the dry consecutive days a balance between the localities was observed. Barra de Guabiraba, Cachoeirinha, Gravatá and Caruaru, presented a decrease of this parameter. As for the maximum amount of precipitation in one day, only the cities of Brejo de Madre de Deus and Cachoeirinha indicated slight negative tendencies not significant, the others indicated decrease. Numbers of days with precipitation above $25 \mathrm{~mm}$ presented negative trends for all cities, but non significant values. Although some of the parameters evaluated do not indicate significant trends, it can be stated that there is a decrease in the amount of rainfall in the region, thus harming any development in the region, mainly agriculture and livestock, which predominates in the region, and that the lack of water makes it impossible to do so.

Keywords: Parameter. Rainfall. Software

\section{INTRODUÇÃO}

A presença de eventos extremos vem sendo cada vez mais evidente em todas as áreas do mundo. São vários os processos que levam a ocorrência de mudanças climáticas, como o aumento das concentrações dos gases como o dióxido de carbono (CO2), metano (CH4) e óxido nitroso(N2) na atmosfera, causando o aumento da temperatura global, elevação do nível dos oceanos e intensificação da variabilidade espacial e da magnitude dos valores de precipitação.

O Quarto Relatório de Avaliação do Grupo de Trabalho I do "Intergovernmental Panel on Climate Change" (IPCC AR4) de fevereiro de 2007, sugeriu que é muito provável, que a 

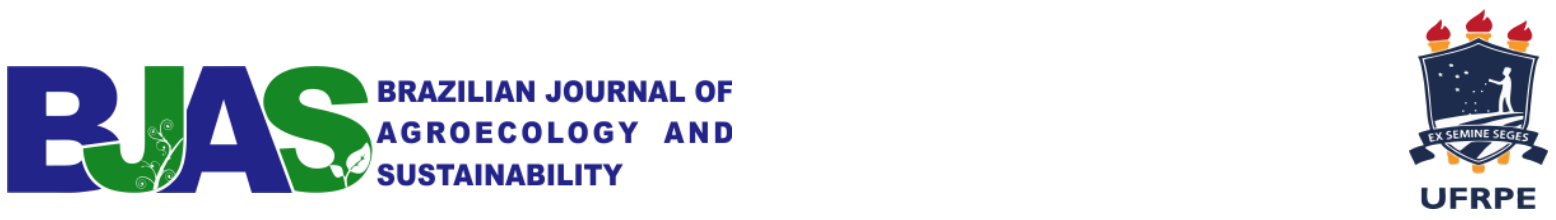

maior parte do aumento na temperatura global média observada no planeta, desde meados do século XX possa ser explicada pelo aumento nas concentrações de gases de efeito estufa (Solomon, 2007), associada às atividades antrópicas. Neste contexto, pode-se observar significativas mudanças no clima e extremos climáticos, e os seus impactos nos sistemas físicos e biológicos devido elevação da temperatura do ar como apresentados no relatório do Grupo de Trabalho II (Solomon, 2007) ).

As projeções futuras para a América Latina mostram que até meados do século XXI, o aumento na temperatura e as reduções de chuva e de vazões de rios poderão levar a grandes alterações em florestas como a floresta Amazônica, por exemplo, e regiões semi-áridass tenderá a ser substituídas por áridas (Salazar, Nobre, \& Oyama, 2007; Solomon, 2007).

Extremos climáticos associados à precipitação pluviométrica afetam diretamente os recursos hídricos, a agricultura e a população; em particular o aumento de perdas econômicas, materiais e até de vidas humanas. (Marengo, Bezerra \& Lacerda, 2011)

Em diversas regiões do Brasil, a ocorrência de eventos extremos é cada vez mais presente. Em regiões áridas e semi- áridas há o risco de desertificação e em regiões como Sul e Sudeste, grandes enchentes até grandes períodos de estiagem, causando assim grandes impactos sociais. Segundo Marengo (2007) o impacto de mudanças climáticas sobre os recursos hídricos no Brasil deverá ser mais dramático no Nordeste, onde a escassez de água é um grande problema. A disponibilidade hídrica per capita na região nos dias atuais é insuficiente nos Estados do Rio Grande do Norte, Paraíba, Pernambuco, Alagoas e Sergipe, além das variações regionais, que tornam a situação ainda mais insustentável para os oito milhões de habitantes do semiárido.

Grande parte da região do semiárido brasileiro enfrenta um problema, já crônico, de falta de água, devido à irregularidade das chuvas e aos baixos índices pluviométricos, principal obstáculo ao desenvolvimento das atividades agrárias e agropecuárias (Marengo, 2011). É uma das regiões mais vulneráveis do País às mudanças e à variabilidade do clima, principalmente aquelas associadas às secas e enchentes. Dessa forma, atividades como agricultura, geração de hidroenergia, saúde e migrações podem ser severamente prejudicadas (Assad \& Pinto, 2008; Centro de Desenvolvimento e Planejamento Regional da UFMG [CEDEPLAR] \& Fiocruz, 2008). 

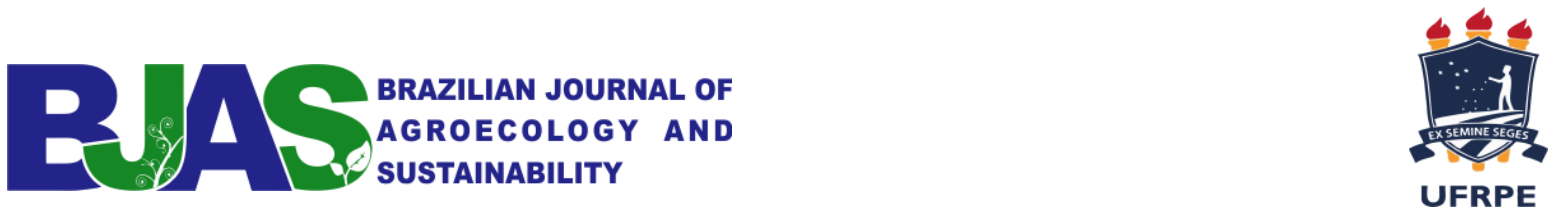

A Embrapa avaliou através de um estudo os impactos que o aquecimento global poderá causar às principais culturas agrícolas do país nas próximas décadas. Segundo esse estudo, “o aquecimento global pode provocar perdas nas safras de grãos de R \$,4 bilhões, já em 2020 o número que pode subir para R \$ 14 bilhões em 2070 e alterar severamente a geografia da produção agrícola no Brasil. Além do mais, a ausência de medidas de mitigação dos efeitos das mudanças climáticas e de adaptação de cultivos pode fazer com que plantações se desloquem para áreas que não se verifica sua ocorrência, porém possuem condições climáticas mais adequadas para se desenvolverem (Empresa Brasileira de Pesquisa Agropecuária [EMBRAPA], 2011).

É característica da região Nordeste o alto potencial para evaporação da água, em função da enorme disponibilidade de energia solar e altas temperaturas. Aumentos de temperatura associados à mudança de clima decorrente do aquecimento global, independente do que possa vir a ocorrer com as chuvas, já seriam suficientes para causar maior evaporação de lagos, açudes e reservatórios e grande evaporação das plantas. Ou seja, se não houver aumento no volume de chuvas, a água se tornará um bem mais escasso, com sérias consequências para a sustentabilidade do desenvolvimento regional. (Marengo, 2010)

Possuindo $24.394 \mathrm{Km}^{2}$, o Agreste, representa uma área de transição entre o litoral e o sertão, situa-se totalmente no planalto da Borborema. Recebem umidade das massas de ar dos sistemas meteorológicos que atuam sobre a mesorregião: Zona de Convergência Intertropical (ZCIT), Ondas de Leste, Vórtice Ciclônico de Atmosfera Superior (VCAS). O Agreste corresponde à bacia leiteira do Estado, e que teve essa posição graças à pecuária sendo uma atividade acessória ao cultivo da cana-de-açúcar na Zona da Mata (Amorim, Santos, \& Ferreira, 2006).

O objetivo do presente trabalho é avaliar as tendências climáticas dependentes da precipitação pluviométrica no Agreste Central de Pernambuco a partir dos índices climáticos, com o propósito de averiguar se houve mudanças no comportamento pluviométrico, uma vez que, para o desenvolvimento de uma região, torna-se importante e necessário o conhecimento de seus recursos naturais.

\section{MATERIAL E MÉTODOS}

\subsection{Caracterização da área de estudo}


A região Agreste de Pernambuco é subdividida em três Regiões de Desenvolvimento (RD's):

Agreste Central, Agreste Meridional e Agreste Setentrional, sendo estas regiões compostas por: 26, 26 e 19 municípios, respectivamente, totalizando 71 municípios (Figura 1).

Figura 1. Mapa de localização do Brasil, com identificação do estado de Pernambuco e destaque para as regiões do Agreste Setentrional, Central e Meridional. Fonte: ITEP (2017), modificado pelos autores.

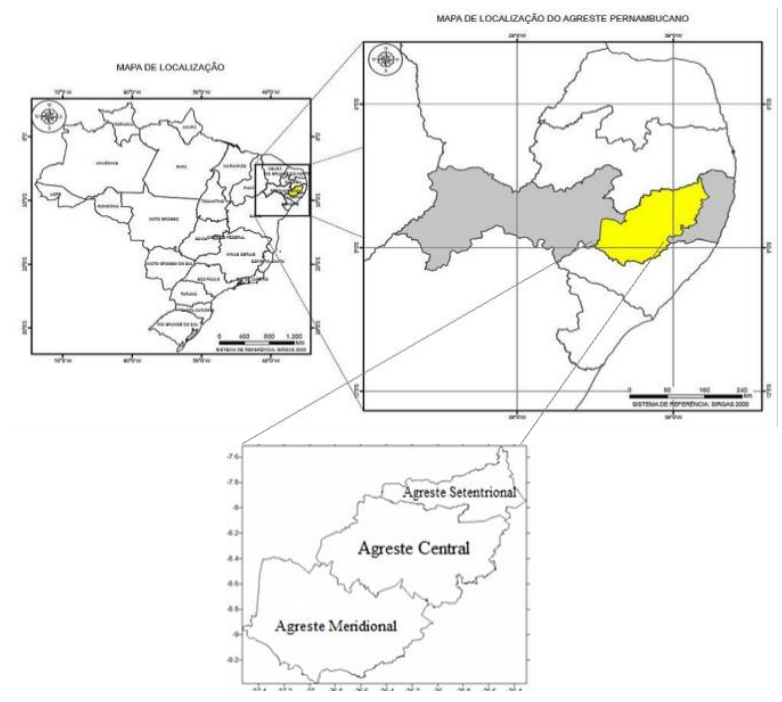

O Agreste central da região do presente estudo, é composto pelos seguintes municípios: Agrestina, Alagoinha, Altinho, Barra de Guabiraba, Belo Jardim, Bezerros, Bonito, Brejo da Madre Deus, Cachoeirinha, Camocim de São Félix, Caruaru, Cupira, Gravatá, Ibirajuba, Jataúba, Lagoa dos Gatos, Panelas, Pesqueira, Poção, Riacho das Almas, Sairé, Sanharó, São Bento do Una, São Caetano, São Joaquim do Monte e Tacaimbó.

\subsection{Material}

Foi realizado o levantamento dos dados mensais de precipitação pluviométrica existentes no período de 1963 a 2017, dos 26 municípios que compreendem a região do Agreste Central de Pernambuco. Após a análise dos dados foram selecionados apenas 9 postos pluviométricos, com séries climatológicas que constituíssem um período de dados maior ou igual a 30 anos. Esses dados das estações pluviométricas foram fornecidos gratuitamente pela Agência 

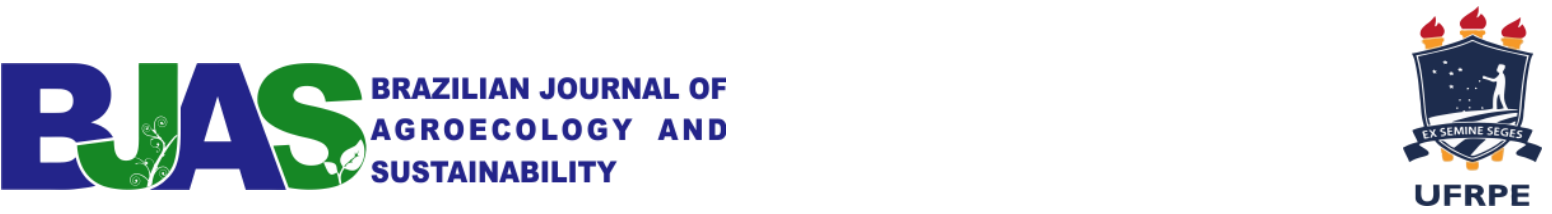

Pernambucana de Águas e Clima (APAC). Na tabela 1 estão as referências dos postos, as cidades e a série de dados de cada posto (início-fim).

É importante ressaltar que muitos desses dados apresentam falhas de informação em determinados períodos, por isso foi necessária a utilização de dados de mais um posto de observação para que a série de informações diárias estivesse mais completa possível.

Quadro 1. Cidades utilizadas no estudo

\begin{tabular}{|c|c|c|}
\hline Referência & Cidade & Início-fim \\
\hline 140 & Alagoinha & $1962-2017$ \\
\hline 58 & Barra de Guabiraba & $1963-2006$ \\
\hline 459 & Barra de Guarabira & $2010-2017$ \\
\hline & Belo Jardim & $1963-2012$ \\
\hline 20 & Bezerros & $2013-2017$ \\
\hline 67 & Brejo da Madre de Deus & $1963-2017$ \\
\hline & Brejo da Madre de Deus & $2013-2017$ \\
\hline 113 & Caruaru & $1962-2012$ \\
\hline & Caruaru & $2013-2017$ \\
\hline 58 & Gravatá & $1933-2017$ \\
\hline 21 & São Bento do Una & $1962-2017$ \\
\hline
\end{tabular}

É importante ressaltar que muitos desses dados apresentam falhas de informação em determinados períodos, por isso foi necessária a utilização de dados de mais um posto de observação para que a série de informações diárias estivesse mais completa possível.

Figura 2. Distribuição espacial das estações pluviométricas na região do Agreste Central. 


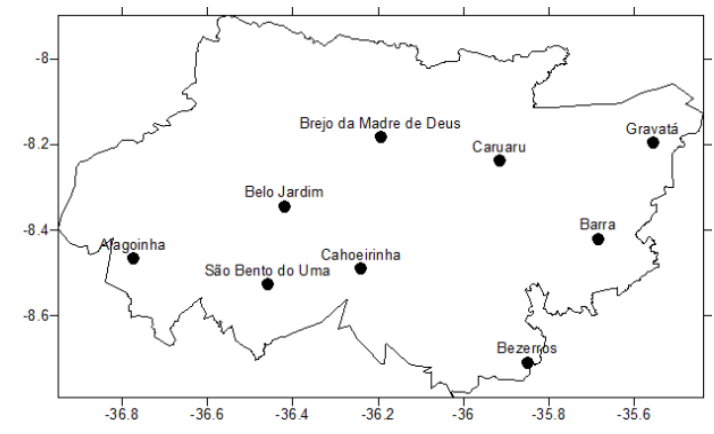

Para o desenvolvimento da pesquisa foi utilizado o software RClimdex que é um programa usado no cálculo de índices de extremos climáticos para monitorar e detectar mudanças climáticas. O RClimdex foi desenvolvido por Byron Gleason do National Climate Data Centre (NCDC) da NOAA, e tem sido usado em oficinas CCI/CLIVAR (International Research Programme on Climate Variability and Predictability) desde 2000. O software calcula todos os 27 índices básicos, derivados da precipitação e da temperatura, recomendados pela equipe de peritos do CCI/CLIVAR para "Climate Change Detection Monitoring and Índices" (ETCCDMI). O pacote RClimdex 2.9.0 é de distribuição gratuita e está disponível no sítio: http://cccma.seos.uvic.ca/ETCCDMI/.

Ressalta-se que dos 27 índices básicos recomendados pela equipe do ETCCDMI (Zhang \& Yang, 2004), serão utilizados quatro índices identificados na Tabela 2, em virtude dos objetivos propostos neste trabalho.

Quadro 2 - Índices climáticos dependentes da precipitação, com suas definições e unidades.

\begin{tabular}{|c|c|c|c|}
\hline ID & Nome do indicador & Definição & Unidade \\
\hline PRCPTOT & $\begin{array}{l}\text { Precipitação total anual nos dias } \\
\text { úmidos }\end{array}$ & $\begin{array}{l}\text { Precipitação total } \\
\text { anual nos dias úmidos } \\
(\mathrm{RR} \geq 1 \mathrm{~mm})\end{array}$ & $\mathrm{mm}$ \\
\hline DCS & Dias consecutivos secos & $\begin{array}{l}\text { Número máximo de } \\
\text { dias consecutivos } \\
\text { secos }(\mathrm{RR}<1 \mathrm{~mm})\end{array}$ & dias \\
\hline Rx1day & $\begin{array}{l}\text { Quantidade máxima de precipitação } \\
\text { em um dia }\end{array}$ & $\begin{array}{l}\text { Máximo } \\
\text { anual de } \\
\text { precipitação } \\
\text { em } 1 \text { dia }\end{array}$ & $\mathrm{mm}$ \\
\hline
\end{tabular}




\begin{tabular}{|l|l|l|c|}
\hline R25mm & Número de dias com precipitação & Número de dias em 1 & dias \\
acima de $25 \mathrm{~mm}$ & em que a & \\
precipitação foi $\geq$ & \\
& & $25 \mathrm{~mm}$ & \\
\hline
\end{tabular}

Fonte: Autor

A seguir estão apresentadas as definições dos 4 índices utilizados nesse trabalho.

1. PRCPTOT (Precipitação total anual nos dias úmidos) [mm]: Seja RRij a quantidade diária de precipitação num dia i num período j . Se I representa o número de dias em então:

$$
\operatorname{PRCPTOT}_{J}=\sum_{i}^{I}=1 R R_{i j}
$$

2. CDD (Dias consecutivos secos) [dias]: Seja RRij a quantidade diária de precipitação num dia i num período j. Conte o maior número de dias consecutivos onde:

$$
R R_{i j}<1 \mathrm{~mm}
$$

3. RX1day (Quantidade máxima de precipitação em um dia) [mm]: Seja RRij o total diário de precipitação num dia i num período j . Então, os valores máximos de 1 dia para o período j são:

$$
R x 1 d a y_{j}=\max \left(R R_{i j}\right)
$$

4. Rnn (Número de dias acima de $n n$ mm) [dias]: Seja RRij a quantidade diária de precipitação num dia i num período j. Se nn representa qualquer valor razoável de precipitação diária, então, conte o número de dias onde:

$$
R R_{i j} \geq n n m m
$$

\subsection{Métodos}

Procurou-se fazer um levantamento o mais preciso possível dos dados climatológicos de precipitação pluviométricas diários de 9 das 71 cidades que compõem o Agreste de estado Pernambuco. 

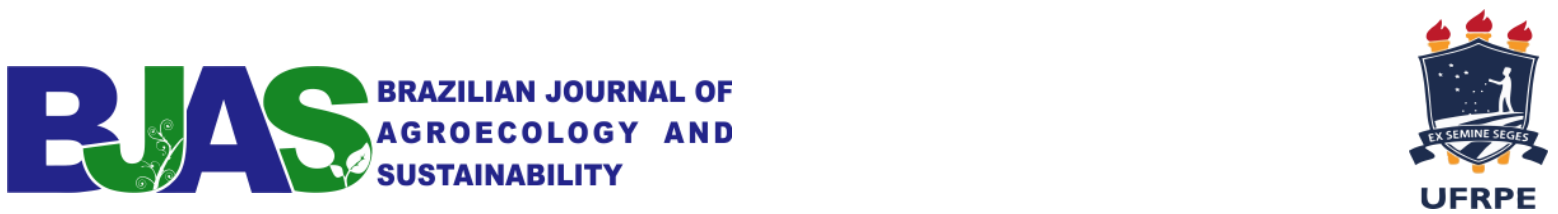

Devido ao fato de todos os postos não apresentarem todos os períodos de informações completos, foram utilizados dados da mesma data e cidade, porém de postos meteorológicos diferentes, para obtenção de uma planilha final de séries de dados diárias utilizada no Rclimdex, e que as falhas nas informações fossem reduzidas ao máximo.

Para a avaliação das tendências foram utilizados os dados corrigidos e padronizados ao máximo para o período de 1963 a 2017.

\section{RESULTADOS E DISCUSSÃO}

Os dados obtidos através do programa serão mostrados a seguir nas figuras 1, 2, 3 e 4, cada uma com seu determinado tipo de avaliação.

Pode-se observar na figura 2 que a maioria das localidades apresentam tendência negativa de Precipitação Total Anual com valores significativos no volume de chuvas, com exceção para Barra De Guabiraba e Cachoeirinha. A cidade de São Bento do Una foi umas das que mostraram uma tendência negativa, porém não muito significante.

Figura 3. Precipitação Total nos dias úmidos nas localidades de Alagoinha (A), Barra de Guabiraba (B), Belo Jardim (C), Bezerros (D), Brejo da Madre de Deus (E), Cachoeirinha (F), Caruaru (G), Gravatá (H) e São Bento do Una (I) no período de 1963 a 2017.
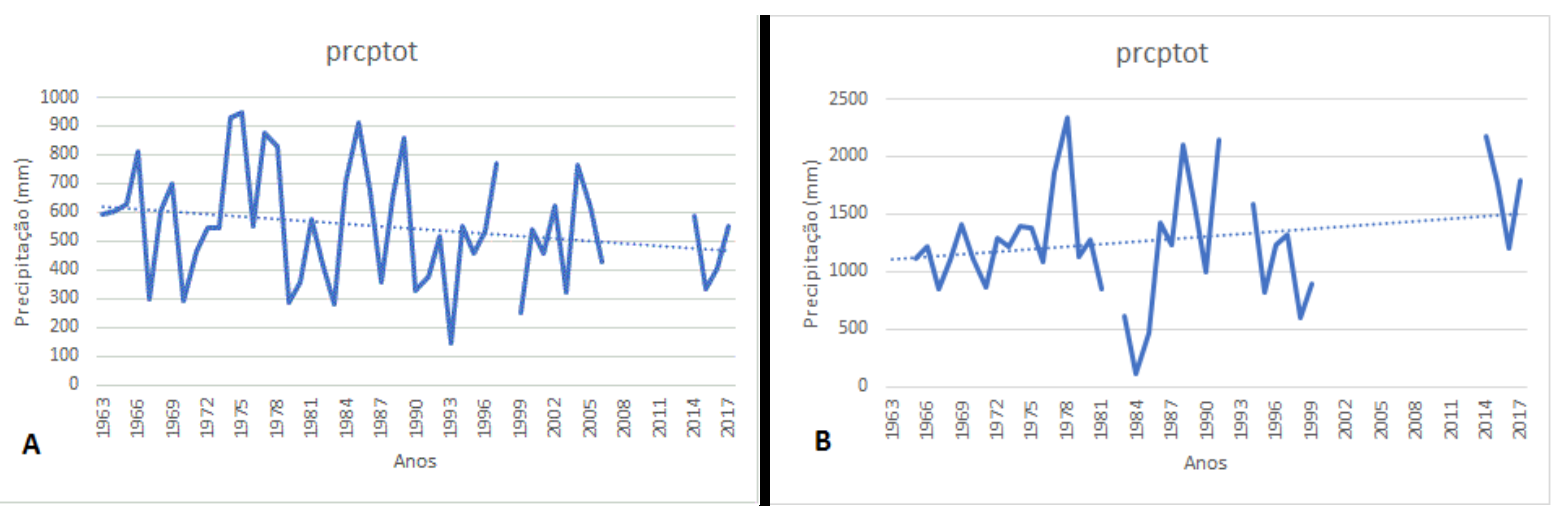

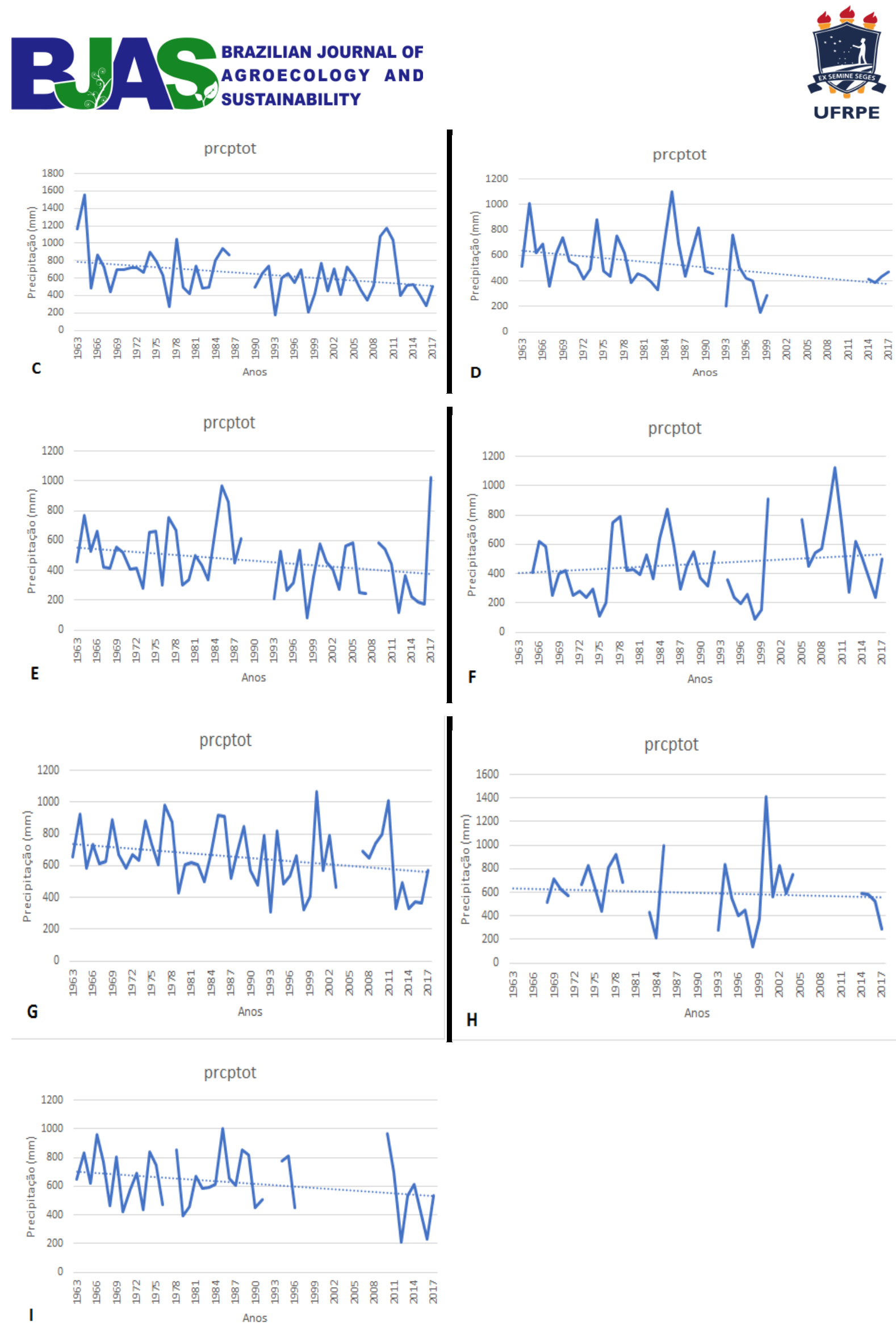

Uma parte dos municípios indicaram um acentuado aumento nos Dias Consecutivos Secos (Figura 3), ou seja, uma maior quantidade de dias com chuva inferior a $1 \mathrm{~mm}$. Contudo, as 

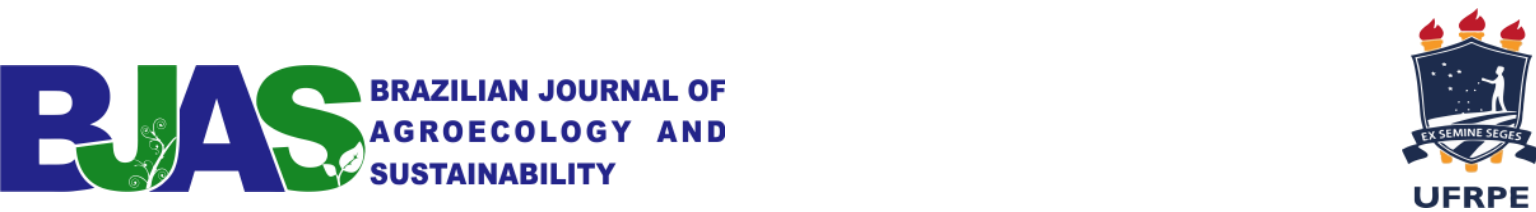

localidades de Barra de Guabiraba, Cachoeirinha, Caruaru e Gravatá apresentaram uma diminuição significativa neste parâmetro.

Figura 4. Número de dias consecutivos secos nas localidades de Alagoinha (A), Barra de Guabiraba (B), Belo Jardim (C), Bezerros (D), Brejo da Madre de Deus (E), Cachoeirinha (F), Caruaru (G), Gravatá (H) e São Bento do Una(I) no período de 1963 a 2017.
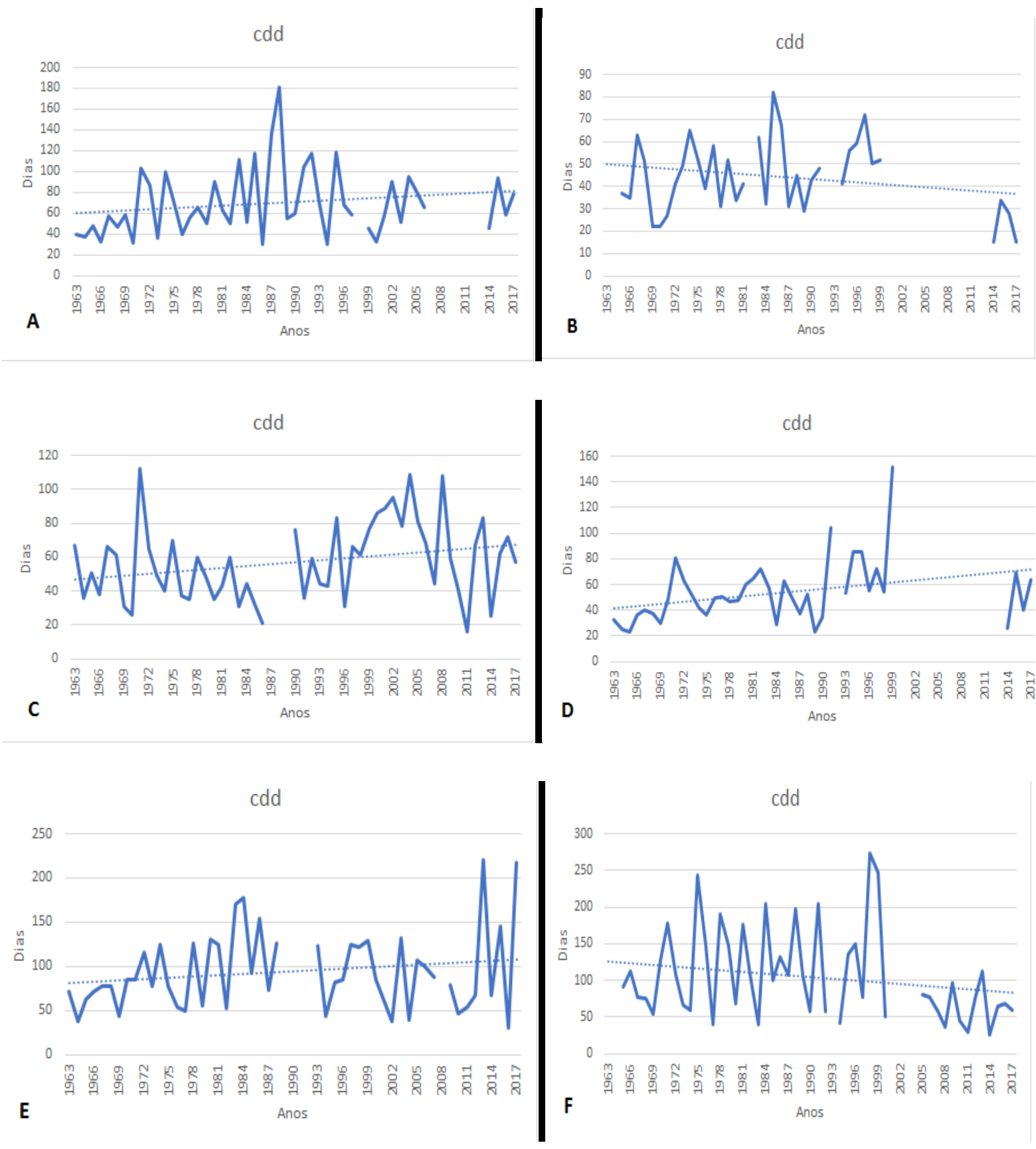

cdd

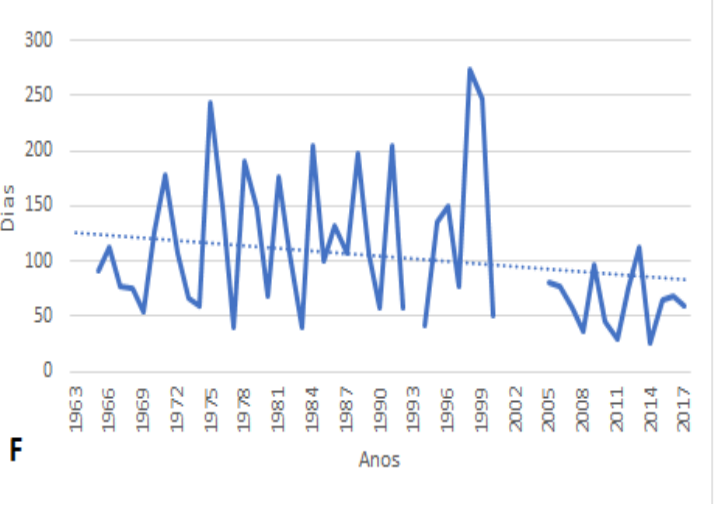



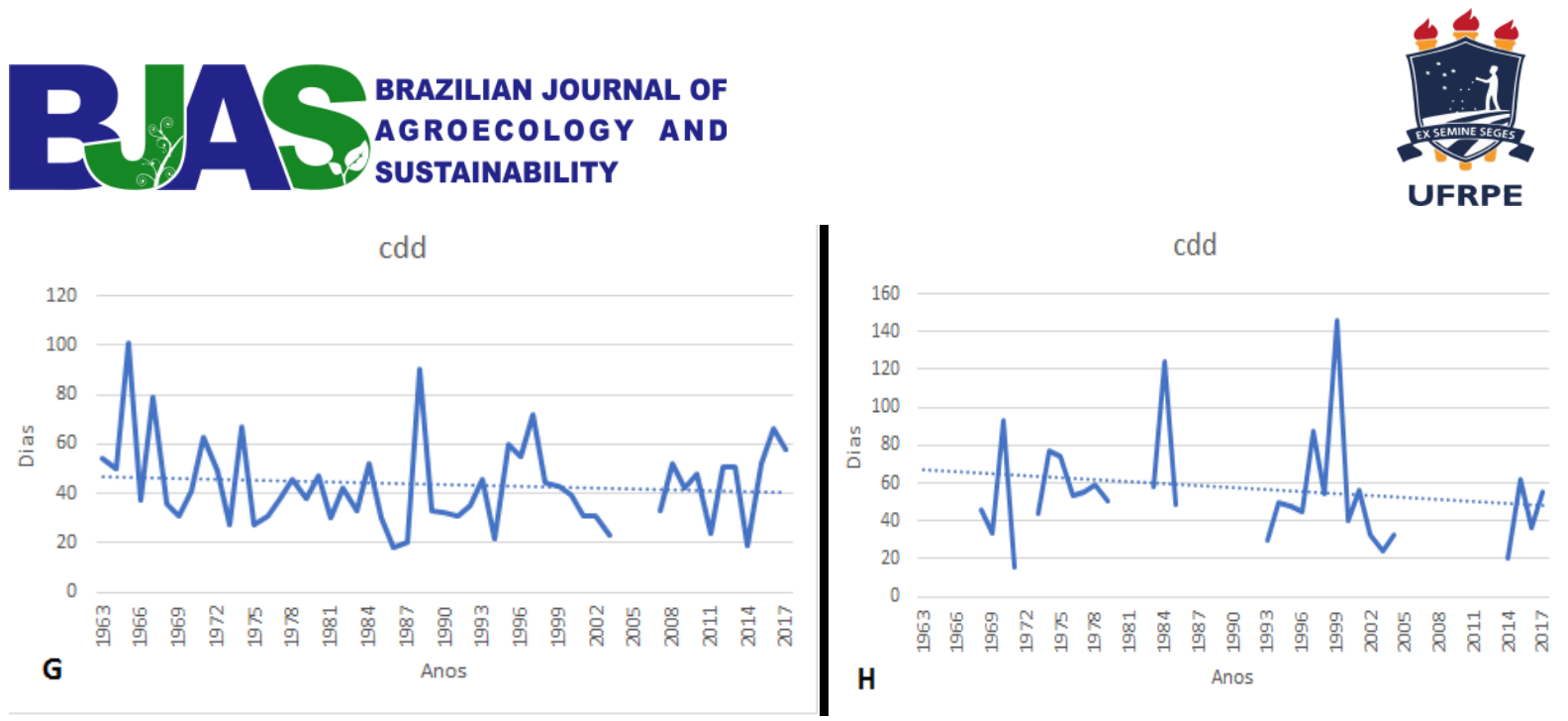

cdd

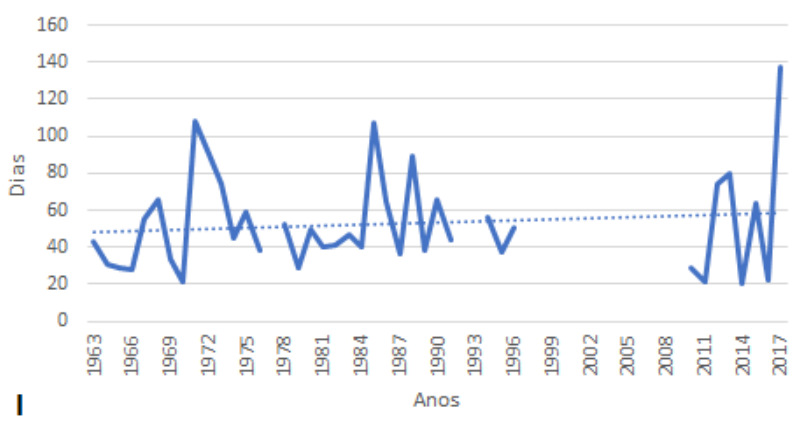

Figura 5. Quantidade máxima de precipitação em um dia de chuva nas localidades de Alagoinha (A), Barra de Guabiraba (B), Belo Jardim (C), Bezerros (D), Brejo da Madre de Deus (E), Cachoeirinha (F), Caruaru (G), Gravatá (H) e São Bento do Una (I) no período de 1963 a 2017.
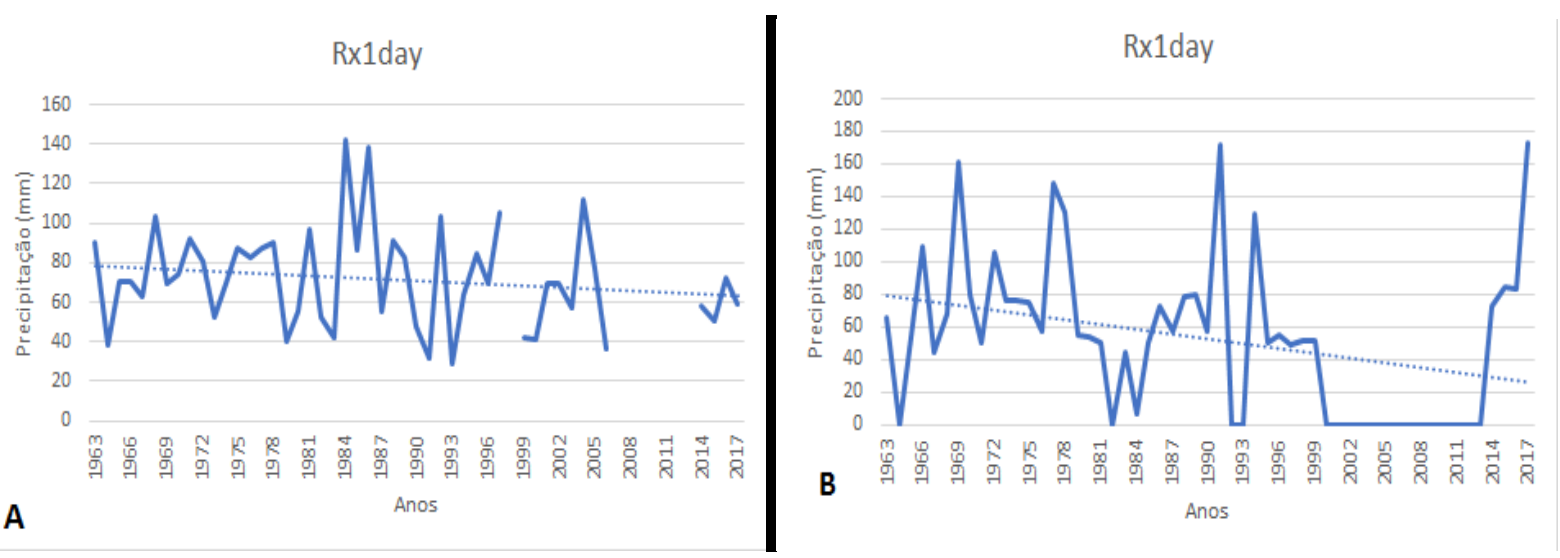


\section{RAG AGROECOLOGY AND SUSTAINABILITY}
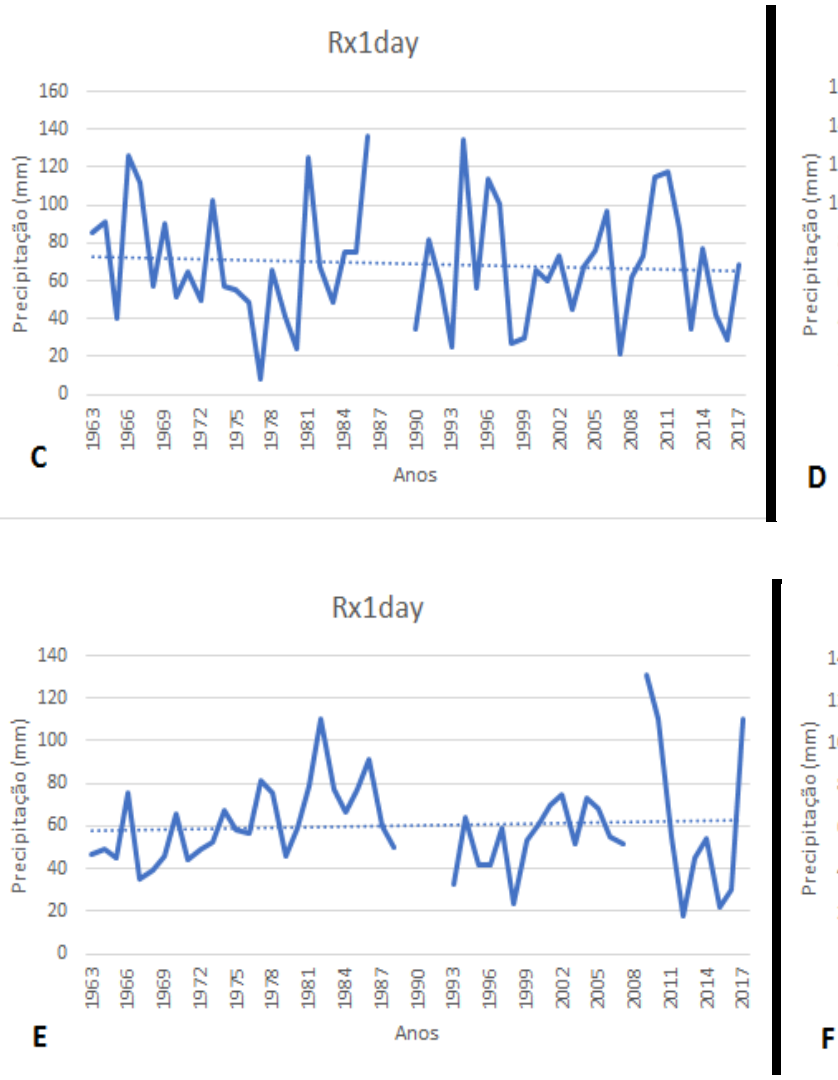

Rx1day

Rx1day
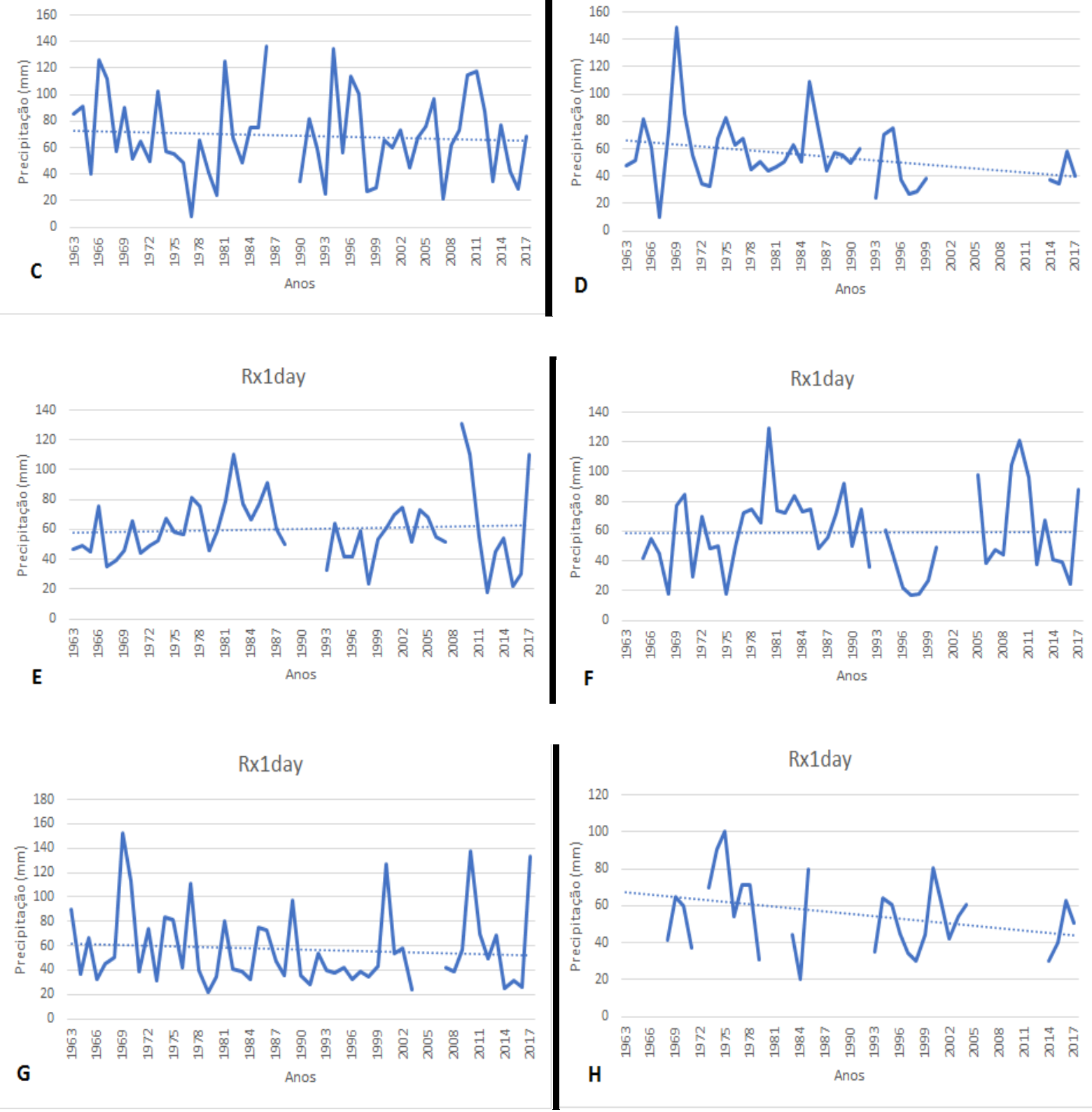

Rx1day

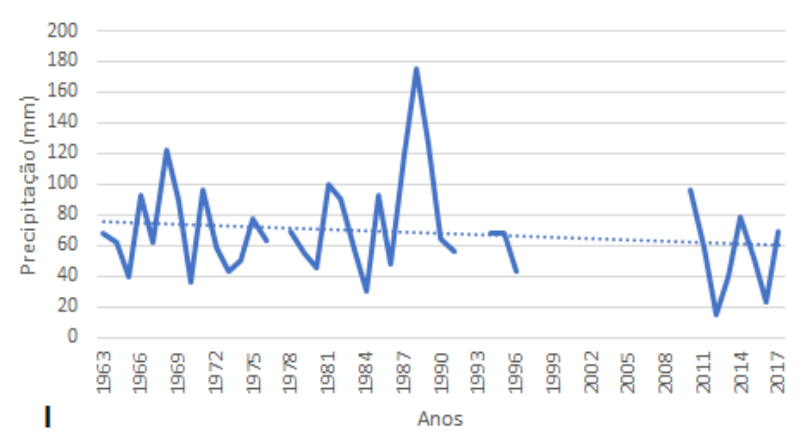



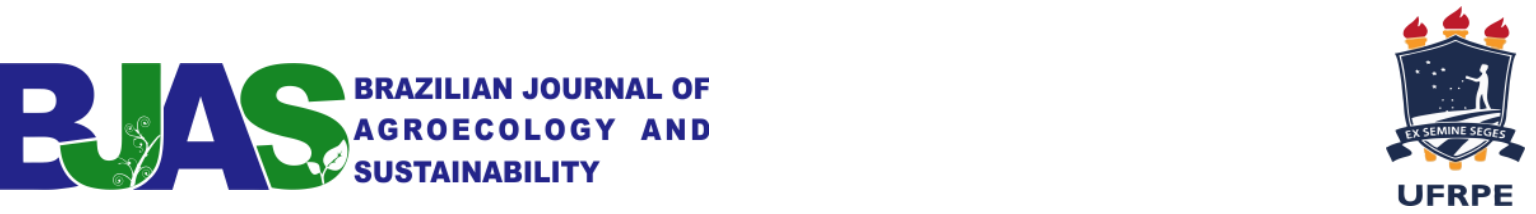

A respeito da Quantidade Máxima de Chuva em Um Dia (Figura 5), com exceção para as cidades de Brejo da Madre de Deus e Cachoeirinha, todas apontaram uma forte diminuição, principalmente, Barra de Guabiraba, Bezerros e Gravatá, com expressivas diminuições.

Figura 6. Número de dias com precipitação acima de $25 \mathrm{~mm}$ nas localidades de Alagoinha (A), Barra de Guabiraba (B), Belo Jardim (C), Bezerros (D), Brejo da Madre de Deus (E), Cachoeirinha (F), Caruaru (G), Gravatá (H) e São Bento do Una (I) no período de 1963 a 2017.
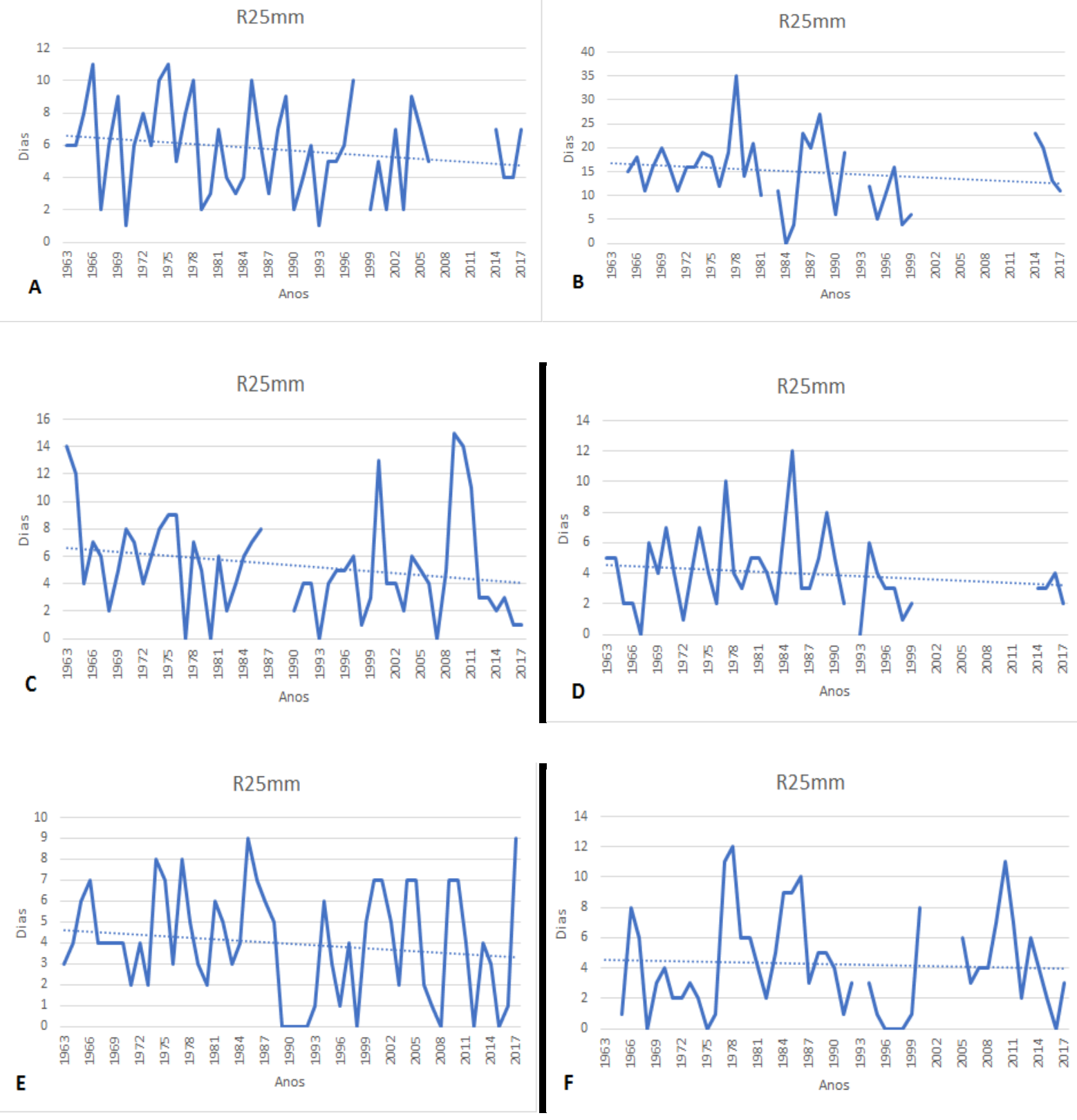

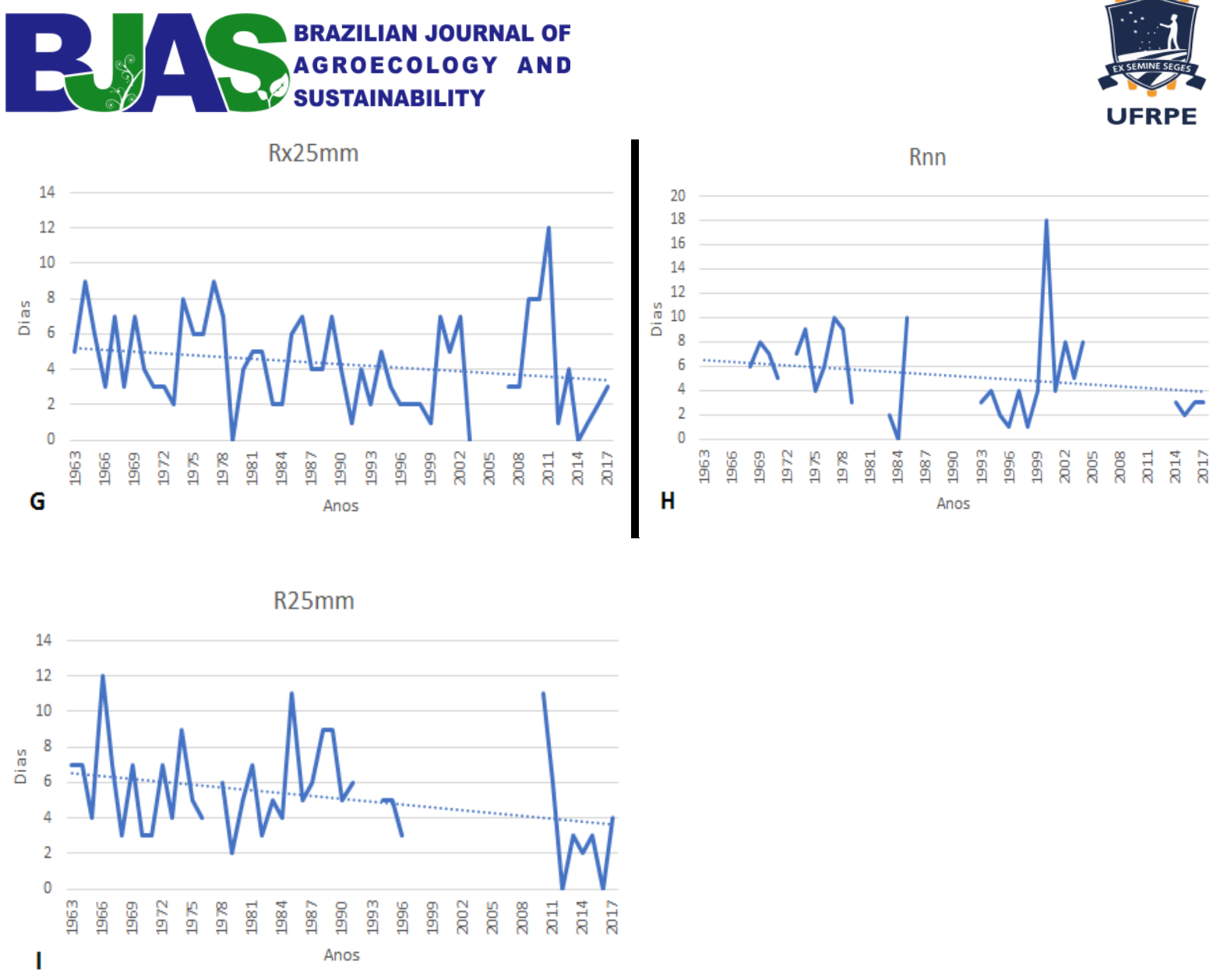

Foi possível constatar que houve uma diminuição na frequência de chuvas acima de $25 \mathrm{~mm}$ (Figura 6), para todas as localidades, mesmo não sendo tendências tão acentuadas. A cidade de Cachoeirinha foi a que não apresentou valores significativos quanto a alteração no seu volume de chuvas.

A saída do RClimdex para todos os índices, fornecem dados estatísticos, tais como: tendência linear calculada pelo método de mínimos quadrados; nível de significância estatística da tendência (valor p); coeficiente de determinação (r2) e erro padrão de estimativa; assim como, os gráficos das series anuais. Foram consideradas como possíveis tendências climáticas os índices que apresentaram tendência linear (positiva ou negativa) superior ao erro padrão de estimativa e estatisticamente significativa $(\mathrm{p}<0,05$ : alta significância estatística; $p<0,1$ : boa significância estatística), obtidos pelo teste “t”" Student, gerado pelo software (Souza \& Azevedo, 2012). 

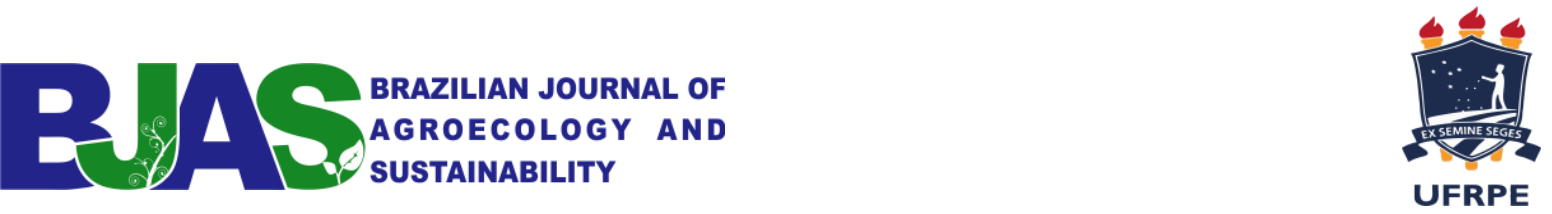

Quadro 3 - Tendências dos índices de extremos climáticos dependentes da precipitação pluvial para o Agreste Central pernambucano. ( $\mathrm{p}<0,05$ : alta significância estatística; $\mathrm{p}<0,1$ : boa significância estatística).

\begin{tabular}{|c|l|l|l|l|}
\hline Municípios & Rx1day & R25mm & \multicolumn{1}{|c|}{ dcs } & prcptot \\
\hline Alagoinha & -0.274 & -0.033 & 0.384 & -2.865 \\
\hline Barra & 0.255 & -0.079 & -0.24 & 7.512 \\
\hline Belo Jardim & -0.136 & -0.047 & $\mathbf{0 . 3 7 2} *$ & $\mathbf{- 4 . 9 9 2 *}$ \\
\hline Bezerros & -0.497 & -0.025 & $\mathbf{0 . 5 4 7} *$ & $\mathbf{- 4 . 7 8 1} *$ \\
\hline Brejo da Madre de Deus & 0.097 & -0.018 & 0.492 & $\mathbf{- 3 . 3 6 1} *$ \\
\hline Cachoeirinha & 0.015 & -0.011 & -0.802 & 2.287 \\
\hline Caruaru & -0.166 & -0.034 & -0.116 & $\mathbf{- 3 . 2 9 2} *$ \\
\hline Gravatá & -0.43 & -0.047 & -0.354 & -1.505 \\
\hline São Bento do Una & -0.303 & $\mathbf{- 0 . 0 5 3} *$ & $\mathbf{0 . 1 8 7} *$ & $\mathbf{- 3 . 2 0 7} *$ \\
\hline
\end{tabular}

Com relação ao Agreste Central (quadro 3), a maioria das localidades apresentaram tendência de diminuição do Rxday e da Precipitação total anual, com exceção da precipitação diária acima de $30 \mathrm{~mm}(\mathrm{R} 30 \mathrm{~mm})$, onde todos os municípios indicam redução dos dias chuvas cima de $30 \mathrm{~mm}$. A maior parte dos municípios analisados apontaram aumento dos dias consecutivos secos (cdd). O município de São Bento do Una destacou-se com maior significância em todos os parâmetros. Ressalta-se que os valores marcados em negrito apresentam significância estatística.

A distribuição espacial dos índices de extremos climáticos no Agreste Central está representada na Figura 7. Apesar da maioria dos parâmetros avaliados não possuírem tendências com significância estatística, observa-se uma diminuição na quantidade de chuva para região e aumento dos dias consecutivos secos.

Figura 7. Distribuição espacial dos índices de extremos climáticos no Agreste Central. 

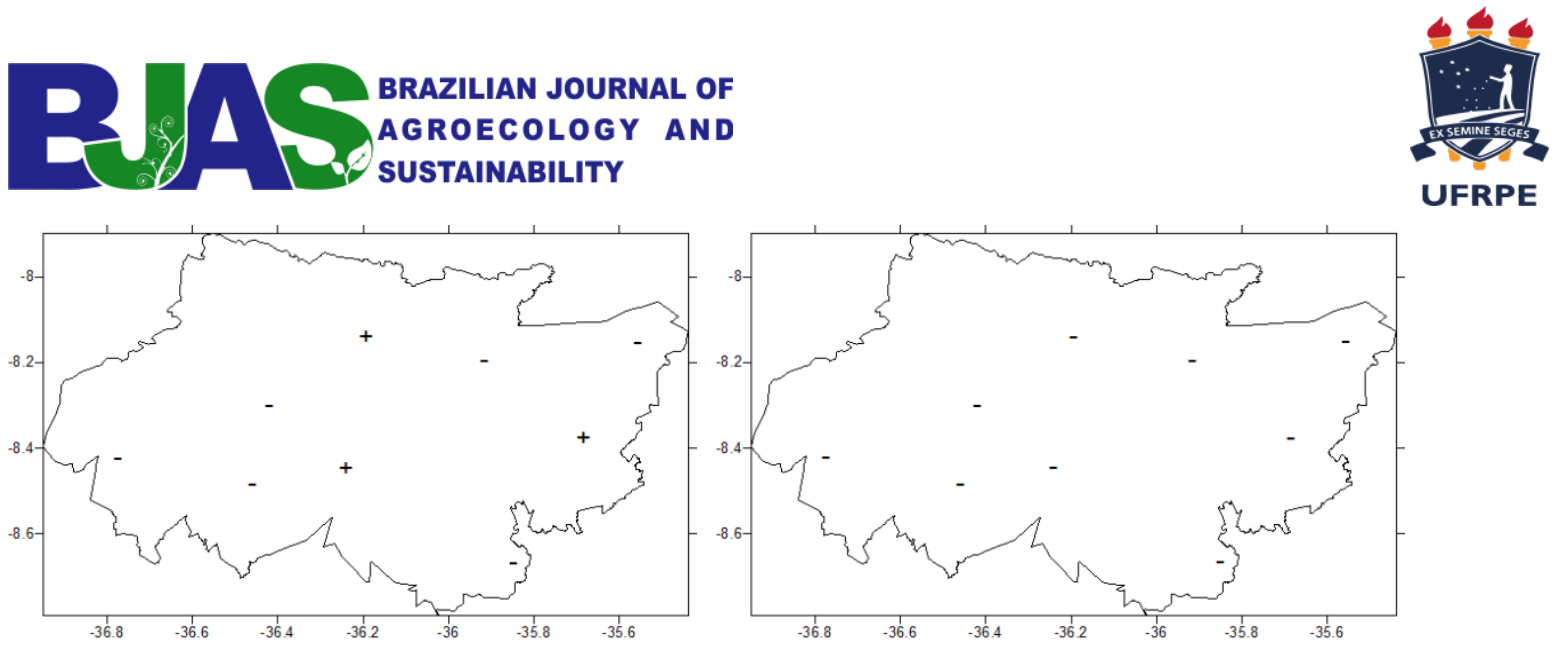

(a) rx1day

(b) R30

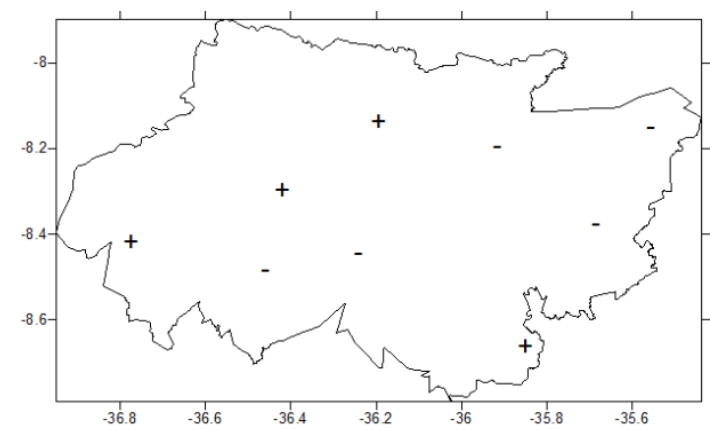

(c)cdd

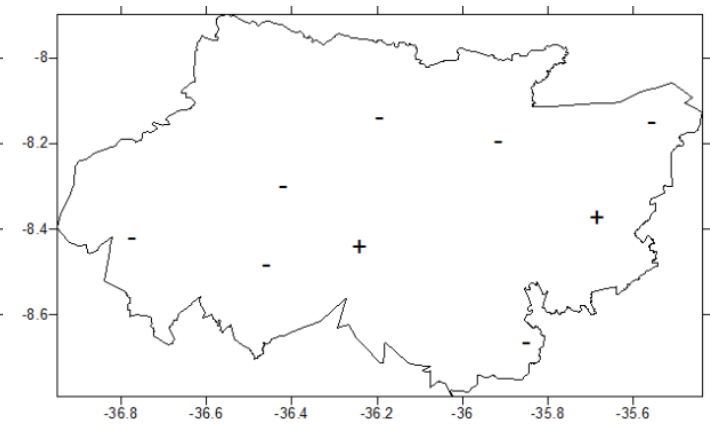

(d) preptot

\section{CONSIDERAÇÕES FINAIS}

Identificou-se diminuição na quantidade de chuva para a região do Agreste Central, redução da precipitação total, de dias com precipitação acima de $25 \mathrm{~mm}$ e aumento da frequência de dias secos.

Apesar dos resultados apontarem oscilações nos parâmetros climáticos, não se deve associar este fato a uma mudança climática global, mas também às ações humanas, onde modifica-se o espaço de diversas formas como, por exemplo, reduzindo áreas verdes, contribuindo para alterações do clima.

\section{REFERÊNCIAS}

Amorim, G. R. S., Santos Júnior, E. A. G., Ferreira, V. S., Carvalho, S. R. P., \& Galvíncio, J. D (2006). Estimativa do balanço hídrico no Agreste de Pernambuco. Anais do Congresso Brasileiro de Meteorologia, Florianópolis, SC, Brasil, 14. 7 p.

Pinto, H. S., \& ASSAD, E. D. (2008). Aquecimento global e cenários futuros da agricultura brasileira. Campinas: Embrapa. 
Cedeplar \& FioCruz. (2008). Mudanças climáticas, migrações e sáude: cenários para o Nordeste brasileiro, 2000-2050. Belo Horizonte: FioCruz/UFMG.

Change, I. C. (2007). The physical science basis. Contribution of Working Group I to the fourth assessment report of the Intergovernmental Panel on Climate Change, 996.

Empresa Brasileira de Pesquisa Agropecuária. (2008). Aquecimento global e a nova geografia da produção agrícola no Brasil. São Paulo. Recuperado de: www.embrapa.br/publicacoes/tecnico/aquecimentoglobal.pdf .

Marengo, J. A., Alves, L. M., Beserra, E. A., \& Lacerda, F. F (2011) Variabilidade e mudanças climáticas no semiárido brasileiro. Recursos hídricos em regiões áridas e semiáridas, 1.

Marengo, J. A. (2007). Mudanças climáticas globais e seus efeitos sobre a biodiversidade: caracterização do clima atual e definição das alterações climáticas para o território brasileiro ao longo do século XXI. Brasília: MMA.

Marengo, J. A. (2010). Vulnerabilidade, impactos e adaptação à mudança do clima no Semiárido do Brasil. Parcerias Estratégicas, 13(27), 149-176.

de Clima, N. M. (2005). Vol. I: Negociações internacionais sobre a mudança de clima; vulnerabilidade, impactos e adaptação à mudança de clima. Cadernos NAE.

Salazar, L. F., Nobre, C. A., \& Oyama, M. D. (2007). Climate change consequences on the biome distribution in tropical South America. Geophysical Research Letters, 34(9).

Solomon, S. (2007, December). IPCC (2007): Climate change the physical science basis. In AGU Fall Meeting Abstracts.

Souza, W. M., \& Azevedo, P. V. (2012). Índices de Detecção de Mudanças Climáticas Derivados da Precipitação Pluviométrica e das Temperaturas em Recife-PE (Detection Indexes Derived from Climate Change Rainfall and Temperatures in Recife-PE). Revista Brasileira de Geografia Física, 5(1), 143-159.

Zhang, X., \& Yang, F. (2004). RClimDex (1.0:) user manual. Ontario, CA: Climate Research Branch Environment Canada Downsview.

\section{AGRADECIMENTOS:}

Os autores agradecem à APAC (Agencia Pernambucana de Águas e Clima) pela concessão dos dados, aos autores do RClimdex (Xuebin Zhang e Feng Yang) por disponibilizarem o "software" gratuitamente. 